\title{
Preparation and Properties of Novel Superhydrophobic Cellulose Nanofiber Aerogels
}

\author{
Guoqing Liu $\mathbb{D}^{1},{ }^{1}$ Jing Li, ${ }^{2,3}$ Xiaodong Li, ${ }^{1}$ Xiangjun Pan, ${ }^{1}$ and Chao Qian ${ }^{2,3}$ \\ ${ }^{1}$ College of Chemical and Material Engineering, Quzhou University, Quzhou, Zhejiang 324000, China \\ ${ }^{2}$ College of Chemical and Biological Engineering, Zhejiang University, Hangzhou, Zhejiang 310027, China \\ ${ }^{3}$ Institute of Zhejiang University-Quzhou, Quzhou, Zhejiang 324000, China
}

Correspondence should be addressed to Guoqing Liu; liuguoqingmr@126.com

Received 23 April 2021; Revised 24 May 2021; Accepted 28 May 2021; Published 15 June 2021

Academic Editor: Arenal Raul

Copyright (c) 2021 Guoqing Liu et al. This is an open access article distributed under the Creative Commons Attribution License, which permits unrestricted use, distribution, and reproduction in any medium, provided the original work is properly cited.

\begin{abstract}
The superhydrophobic cellulose nanofiber aerogels were prepared via sol-gel and subsequent freeze-drying with cellulose nanofibers as raw materials and perfluorohexyl ethyl trimethoxysilane and 3-aminopropyl trimethoxysilane as modifying monomers. The effect of volume ratio and total dosage of the two modifying monomers on the superhydrophobic properties was investigated, and the property variations of the cellulose nanofibers before and after modification were also characterized by FT-IR, XRD, TGA, SEM, XPS, and laser flash diffusivity apparatus. The results showed that the modifying monomers were successfully grafted onto cellulose nanofibers, and the prepared modified cellulose nanofiber aerogels had higher thermal stability. After modification, a micron-level arrayed three-dimensional grid superhydrophobic surface structure was constructed, and the surface energy was reduced. The prepared aerogels exhibited superhydrophobicity with a water contact angle up to $151^{\circ}$ and excellent thermal insulation performance with a thermal conductivity of $0.035 \mathrm{~W} \cdot \mathrm{m}^{-1} \cdot \mathrm{K}^{-1}$, which displayed promising application potential in the field of thermal insulation and waterproof materials.
\end{abstract}

\section{Introduction}

Cellulose is one of the most abundant renewable and degradable biomass resources in nature. Currently, processing it into nanocellulose is one of the most important ways to develop its high-value applications [1-4]. Nanocellulose has many excellent properties such as large specific surface area, high modulus, high strength, high thermal stability, degradability, and biocompatibility, as well as special optical properties and rheological properties. According to its morphological characteristics and aspect ratio, it can be divided into two categories: cellulose nanofiber (CNF) and cellulose nanocrystal (CNC) [1, 5-7]. However, due to the presence of a large number of hydroxyl groups on the surface, nanocellulose has relatively high surface energy and is prone to agglomeration. In addition, the higher hydrophilicity of nanocellulose leads to the poor interface compatibility with weak polar solvents, polymer media, and other systems, which makes it unable to show its excellent performance and seriously impeding its applica- tion in various fields. In order to expand its application in paper industry, energy storage materials, waste water treatment, composite coatings, daily chemical industry, petroleum exploration, and fluid modification [1], it is necessary to modify its chemical structure and charge state of nanocellulose to obtain new functional nanocellulose materials via chemical methods $[8,9]$.

Nowadays, the preparation of nanocellulose-based superhydrophobic aerogel materials has attracted increasing attention in the field of nanocellulose. The imparting of superhydrophobic properties mainly origins from two aspects. One is to construct a micro-nano surface structure, and the other is to reduce the surface energy $[10,11]$. The most widely reported modification method was silanization modification [11-16], generally by using fluorinecontaining silane, fluorine-containing chlorosilane, and silane coupling agent as modifying monomers [16-19], and the reaction was performed by using organic solvent as a dispersion medium. Chemical vapor deposition was also used to prepare superhydrophobic nanocellulose [20]. 
However, these preparation methods have some disadvantages, such as complicated process, high cost of raw materials, and safety and environment problems. Inspired by the above considerations, we reported here cost-effective 3-aminopropyl trimethoxysilane and perfluorohexyl ethyl trimethoxysilane were used to modify the nanocellulose by sol-gel method in which the water was used as the only solvent, and then, the superhydrophobic cellulose nanofiber aerogel (SH-CNF) was obtained by freeze-drying method.

\section{Experimental Section}

2.1. Instrument and Reagent. The instruments used are as follows: ultrasonic cleaner (KQ-100, Kunshan Ultrasonic Instrument Co., Ltd.); hotplate stirrer for round-bottomflasks (DHT, Shandong Hualu Electric Heating Instrument Co., Ltd.); freeze dryer (LC-10N-50A, Zhejiang Lichen Instrument Technology Co., Ltd.); infrared spectrometer (Nicolet Is50, Thermo Fisher Scientific); field emission scanning electron microscope (SU8010, Hitachi Co., Ltd.); contact angle meter (DSA30S, Kruss Scientific Instruments Co., Ltd.); thermal gravimetric analyzer (STA8000, PerkinElmer Instruments Co., Ltd.); X-ray diffractometer (XRD-6100, Shimadzu Corporation); X-ray photoelectron spectrometer (K-Alpha, Thermo Fisher Scientific); and laser flash diffusivity apparatus (LFA 467, NETZSCH Manufacturing Co., Ltd.).

The reagents are as follows: cellulose nanofiber (CNF, $1 \mathrm{wt} \%$, width: $10-80 \mathrm{~nm}$, length: $800-3000 \mathrm{~nm}$ ) was provided by Jinjiahao Green Nanomaterials Co., Ltd.; perfluorohexyl ethyl trimethoxysilane (PFOTMS, 97\%) and 3-aminopropyl trimethoxysilane (KH540, 97\%) were provided by Shanghai Macklin Biochemical Technology Co. Ltd.

2.2. Preparation of SH-CNF. $100 \mathrm{~mL} 1 \mathrm{wt} \% \mathrm{CNF}$ emulsion was placed in a beaker and dispersed under ultrasonic for $30 \mathrm{~min}$. And then, the emulsion was transferred into a $250 \mathrm{~mL}$ three-necked flask which was put in a hotplate stirrer for round-bottom-flasks. The reaction temperature was gradually increased to $50^{\circ} \mathrm{C}$ under magnetic stirring. Meanwhile, $0.8 \mathrm{~mL}$ PFOTMS and $1.2 \mathrm{~mL}$ KH540 were added dropwise. The mixture was kept stirred for $6 \mathrm{~h}$. After completion, the reaction mixture was cooled to room temperature. The final superhydrophobic cellulose nanofiber aerogel sample was obtained with vacuum freeze-drying procedure for $24 \mathrm{~h}$, which was stored in a desiccator for further using.

\section{Results and Discussion}

3.1. Effect of the Volume Ratio of Two Modifying Monomers on Superhydrophobic Property. The volume ratio of the modifying monomers to the raw materials CNF was determined to be $2 \%$, while the volume ratio of the two modifying monomers was also adjusted (Table 1). A series of samples were prepared according to the aforementioned method, and the static-state contact angle of the water droplet was determined; the results were shown in Figure 1. It was found that when CNF was only modified with KH540, the contact angle of the prepared sample was only $77^{\circ}$, and there was no hydrophobicity. However, as the dosage of PFOTMS gradually
TABLE 1: The dosages of modifying monomers.

\begin{tabular}{lcc}
\hline PFOTMS $(\mathrm{mL})$ & KH540 $(\mathrm{mL})$ & PFOTMS : KH540 $(v / v)$ \\
\hline 0 & 2 & $0: 2$ \\
0.4 & 1.6 & $1: 4$ \\
0.8 & 1.2 & $2: 3$ \\
1.2 & 0.8 & $3: 2$ \\
1.6 & 0.4 & $4: 1$ \\
2 & 0 & $2: 0$ \\
\hline
\end{tabular}

increased, as well as the volume ratio of PFOTMS: KH540 $(v / v)$ increased, the contact angle showed an obvious increase, and the hydrophobicity of the sample was enhanced. When PFOTMS:KH540 $(v / v)$ was 2:3, the contact angle of the prepared sample was $151^{\circ}$, obtaining a superhydrophobic state. When PFOTMS : KH540 ( $v / v)$ was $3: 2$, the sample still maintained a superhydrophobic state, but the contact angle did not increase significantly. When the dosage of PFOTMS continued to increase, the contact angle and hydrophobic properties of the prepared sample conversely decreased. When CNF was only modified with PFOTMS, the contact angle of the sample dropped to $120^{\circ}$. This is mainly due to the continuous increase of the dosage of PFOTMS, surface energy of the prepared sample decreased continuously, and the hydrophobic property was enhanced. When PFOTMS: KH540 $(v / v)$ was $2: 3$ (or 3:2), the surface energy of the prepared sample was basically reduced to the minimum; a balance between the surface energy and the micro-nano structure was achieved, thus showing a superhydrophobic state. When the dosage of PFOTMS continues to increase, the surface energy still remains the minimum, but the surface micro-nano structure is damaged, which shows the decline of contact angle and hydrophobic property. Therefore, considering the hydrophobic property and cost, the volume ratio of the modified monomers was determined as PFOTMS : $\mathrm{KH} 540(v / v)=2: 3$.

3.2. Effect of the Total Dosage of the Two Modifying Monomers on Superhydrophobic Property. The total dosage of the two modifying monomers was adjusted $(1,1.5,2,2.5$, and $3 \mathrm{~mL}$ ) for comparison tests with volume ratio of PFOTMS : KH540=2 : 3 . A series of samples was then prepared, and the contact angle of water droplet was determined (as shown in Figure 2). It could be seen that as the total dosage of the two monomers increased, the contact angle of the sample continued to increase, and the hydrophobic performance was enhanced. When the total dosage of the two monomers was $2 \mathrm{~mL}$, the sample reached a superhydrophobic state, and the contact angle was over $150^{\circ}$. At this point, when total dosage of two modifying monomers increased, contact angle of the sample showed negligible changes. This is mainly due to the existence of steric hindrance on the surface of CNF; the hydroxyl groups which could be modified by monomers has a saturation value, which is consistent with the fact that only a part of the hydroxyl groups of CNF could be substituted. Therefore, the total dosage of the two modifying monomers was determined to be $2 \mathrm{~mL}$. 


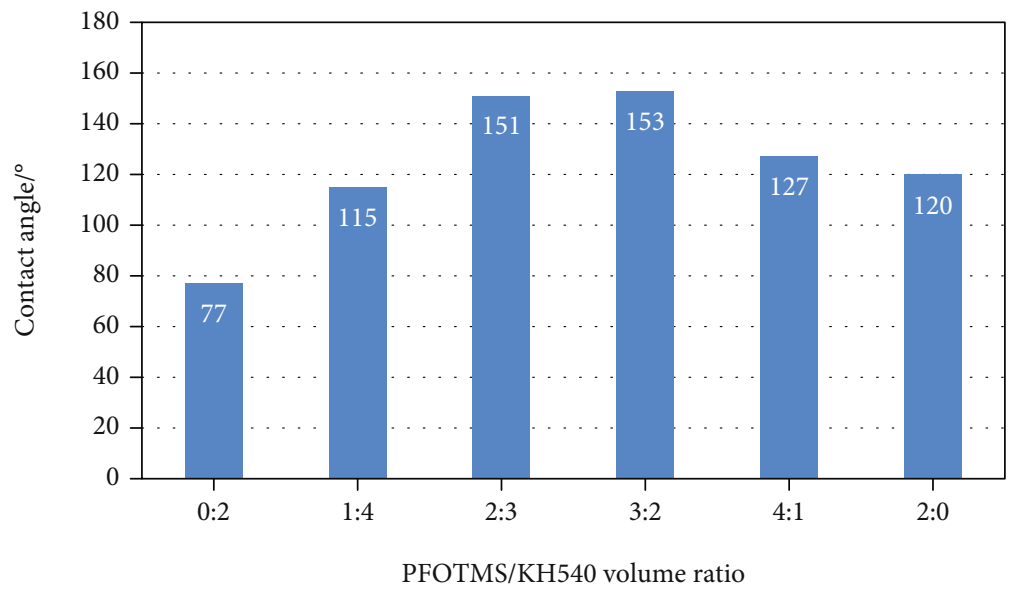

FIgURE 1: Contact angle of water droplet as a function of PFOTMS/KH540 volume ratio.

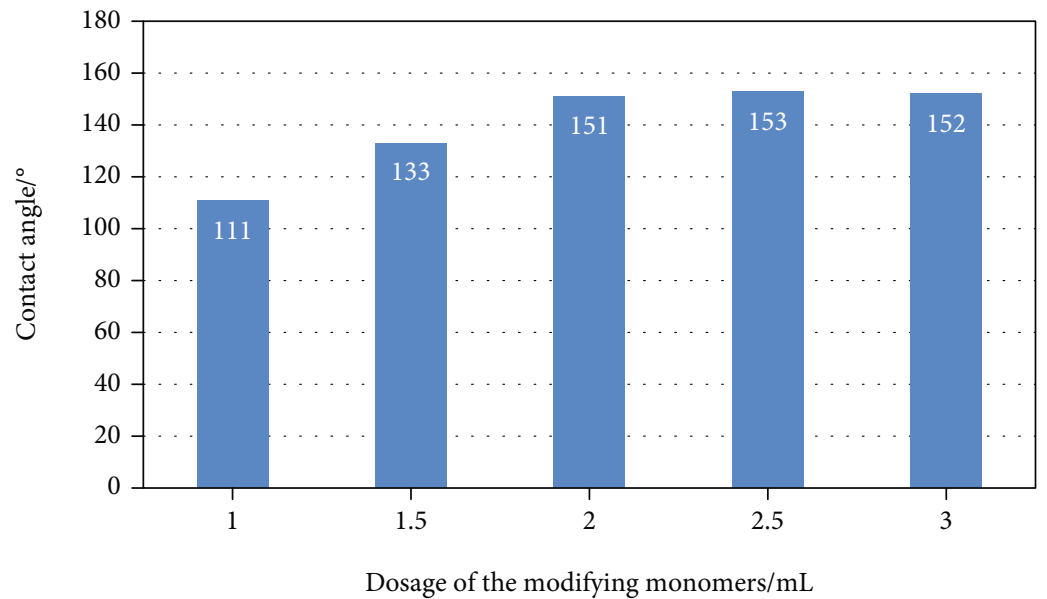

FIGURE 2: Contact angle of water droplet as a function of dosage of the modifying monomers.

3.3. Superhydrophobic Property. The prepared modified cellulose nanofiber aerogels were used for dripping application experiments with aqueous red-ink solution (as shown in Figure 3). It could be seen that the water droplet basically maintained a spherical state and stood on the surface of modified cellulose nanofiber aerogels. The static contact angle of the water droplet was measured to be $151^{\circ}$, which exhibited superhydrophobic property. Therefore, the use of cheap KH540 combined with PFOTMS to modify cellulose nanofibers can obtain superhydrophobic property and achieve the goal of reducing cost and simplifying the reaction process to prepare superhydrophobic cellulose nanofiber aerogels.

3.4. FT-IR. FT-IR was used to characterize the functional group changes of CNF and SH-CNF, and the results were shown in Figure 4. It could be seen that both CNF and SHCNF spectra showed a large broad peak at the wavenumber of $3335 \mathrm{~cm}^{-1}$, assigned to the stretching vibration of hydroxyl groups. Compared to that of CNF, SH-CNF showed a weaker peak intensity, indicating that some hydroxyl groups were substituted by the modifying monomers. The characteristic peak at the $2907 \mathrm{~cm}^{-1}$ was caused by the symmetric stretch-

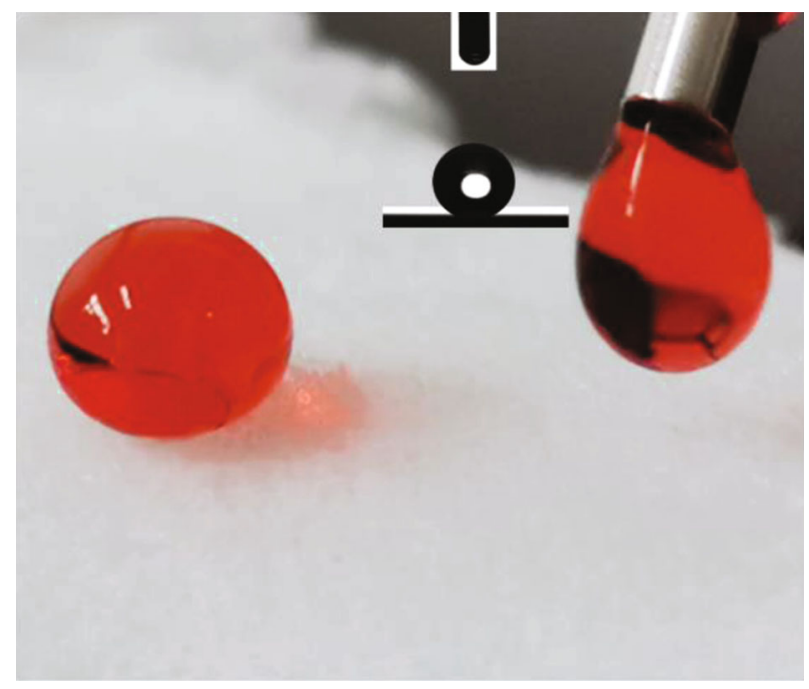

FIgURe 3: Digital image of contact angle test and superhydrophobic performance. 


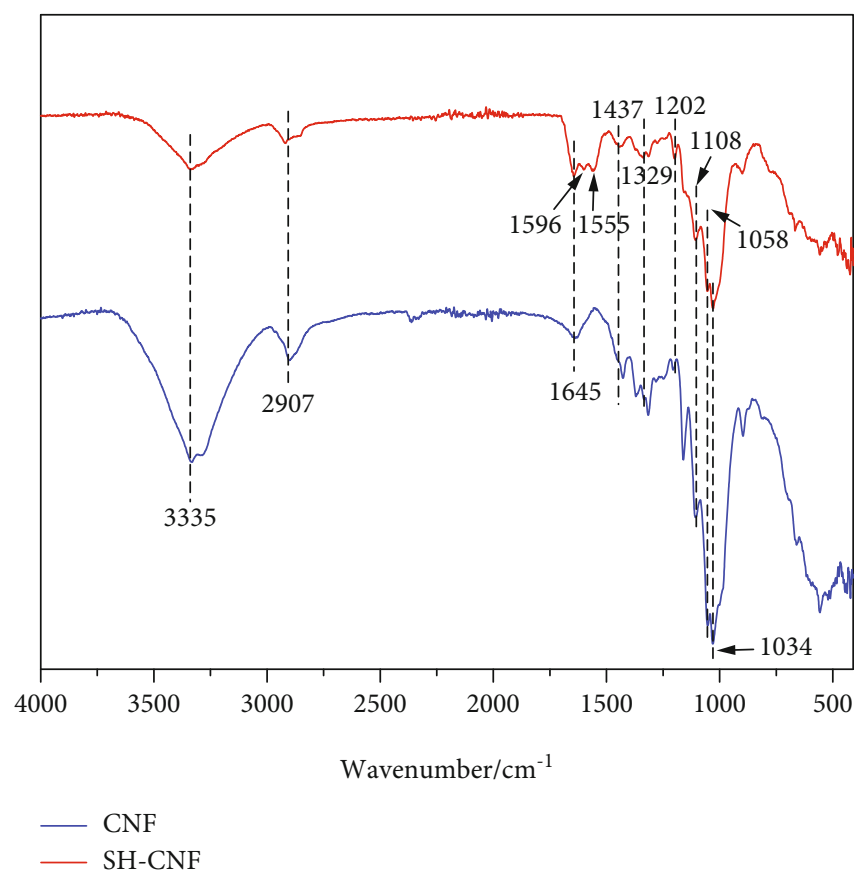

FIGURE 4: FT-IR spectra of CNF and SH-CNF.

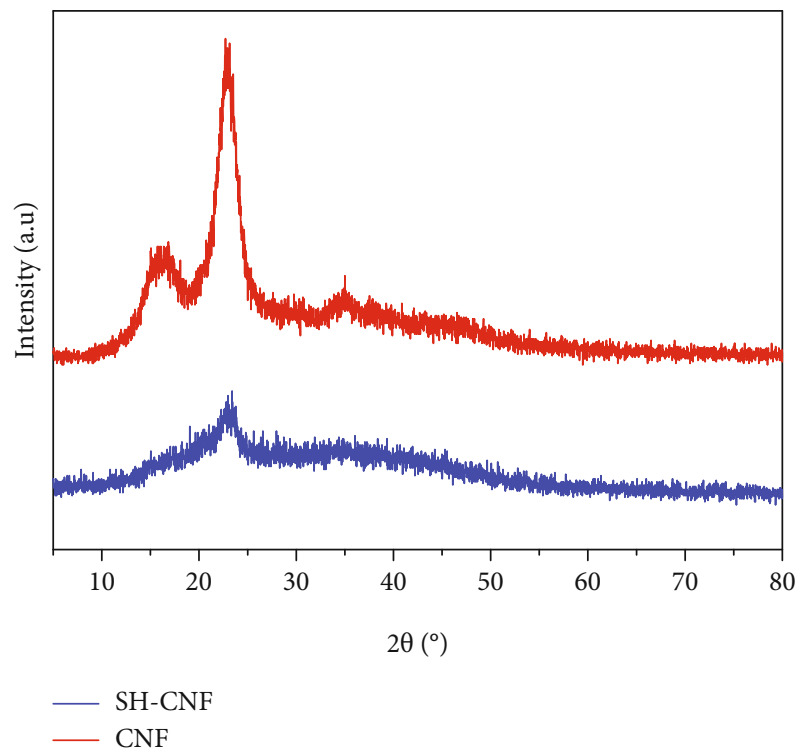

Figure 5: XRD patterns of CNF and SH-CNF.

ing vibration of the $\mathrm{C}-\mathrm{H}$ bond. The characteristic absorption peaks appearing at $1596 \mathrm{~cm}^{-1}$ and $1555 \mathrm{~cm}^{-1}$ were ascribed to the bending vibration peaks of $\mathrm{NH}_{2}$ of $\mathrm{KH} 540$. The peaks at $1437,1329,1202,1108$, and $1058 \mathrm{~cm}^{-1}$ were resulted from the stretching vibration of $\mathrm{CF}_{3}, \mathrm{CF}_{2}$, and $\mathrm{Si}-\mathrm{O}-\mathrm{Si}$ of the modifying monomers PFOTMS and KH540. For CNF, peaks at $1000-1450 \mathrm{~cm}^{-1}$ were resulted from the stretching vibration of $\mathrm{C}-\mathrm{C}$ and $\mathrm{C}-\mathrm{OH}$ and the bending vibration of saturated $\mathrm{C}-\mathrm{H}$ and $-\mathrm{OH}$ among $\mathrm{CNF}$ framework. The characteristic peaks of CNF and SH-CNF at $1000-1450 \mathrm{~cm}^{-1}$ partially overlapped, and the characteristic peak at $1034 \mathrm{~cm}^{-1}$

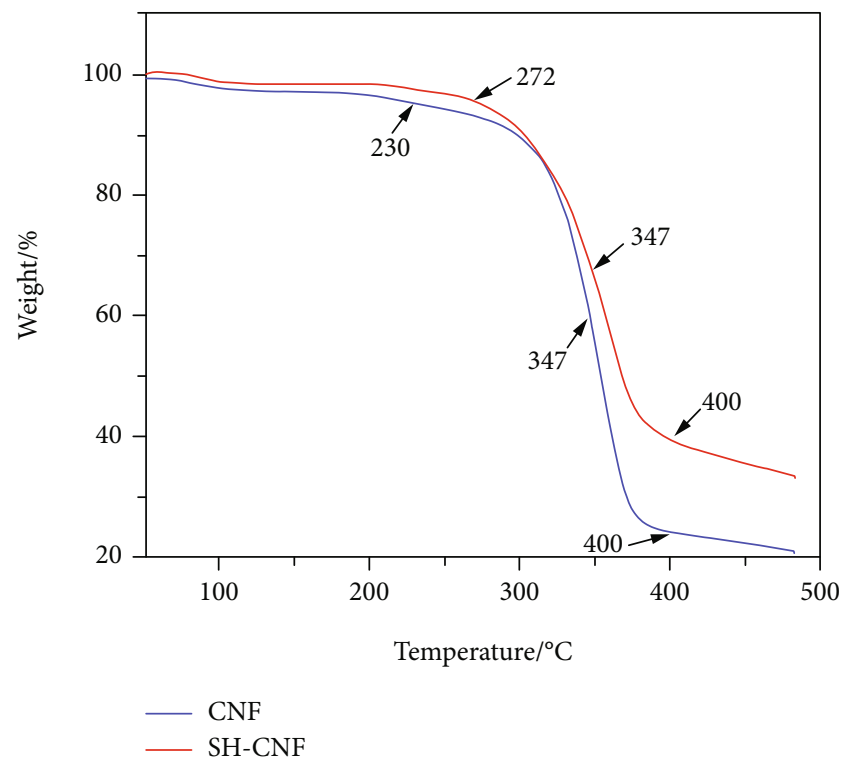

Figure 6: TGA curves of CNF and SH-CNF.

was attributed to the stretching vibration absorption peak of $\mathrm{C}-\mathrm{O}-\mathrm{C}$ among the cellulose nanofiber skeleton. The above results indicated that parts of the hydroxyl groups in CNF were modified by PFOTMS and KH540, but the original main structure remains unchanged.

3.5. X-Ray Diffraction (XRD). The crystalline structure of cellulose nanofiber (CNF) and superhydrophobic cellulose nanofiber ( $\mathrm{SH}-\mathrm{CNF}$ ) was characterized by XRD (Figure 5). It could be seen that the CNF was ascribed to cellulose type I, and the characteristic diffraction peaks at $2 \theta=15.0^{\circ}$, 


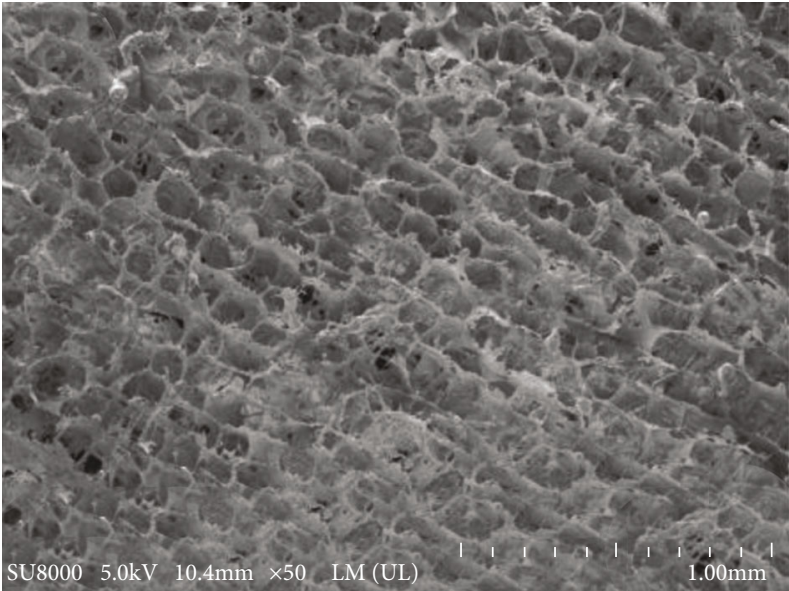

(a)

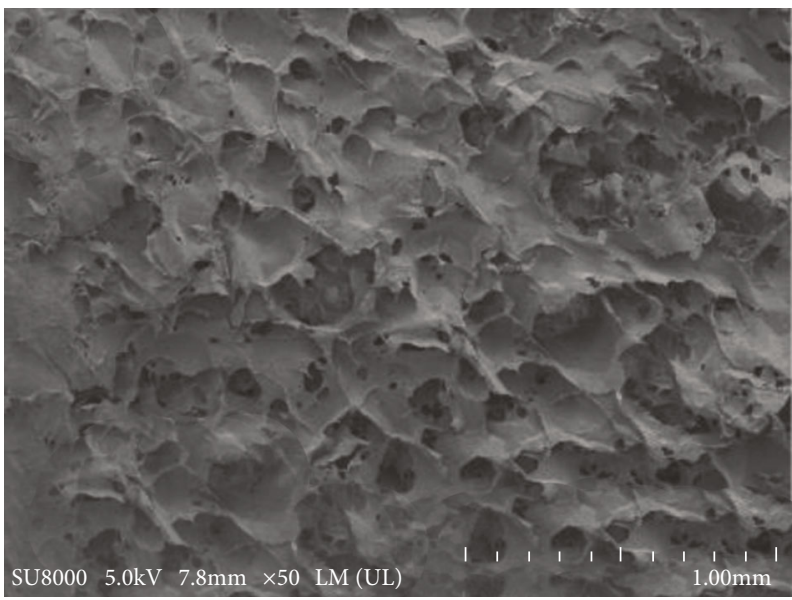

(c)

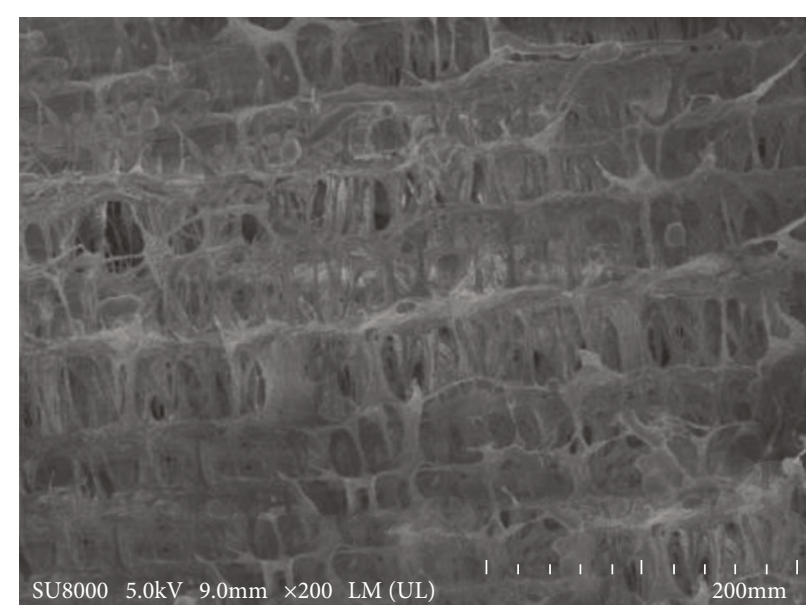

(b)

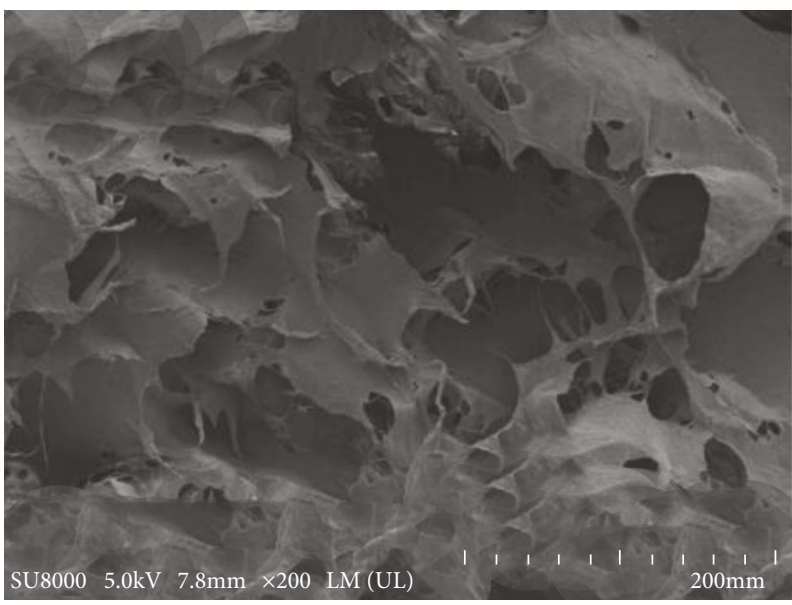

(d)

Figure 7: SEM images of (a,b) SH-CNF and (c, d) CNF.

$16.7^{\circ}, 23.0^{\circ}$, and $35.2^{\circ}$ were observed, and the strongest diffraction peak at $23.0^{\circ}$ was assigned to (002) crystal plane. However, the modified SH-CNF was assigned to type II, and the characteristic diffraction peaks at $2 \theta=15.0^{\circ}, 16.7^{\circ}$, and $35.2^{\circ}$ basically disappeared, and the intensity of the characteristic diffraction peak at $23.0^{\circ}$ was significantly decreased. These results indicated that the modifying monomers PFOTMS and KH540 had reacted with hydroxyl groups on the surface of the CNF crystal structure, accompanied with some cross-linking reactions, which destroyed the crystal structure of CNF $[21,22]$. Based on the Scherrer formula, it could be speculated that the grain size of the modified $\mathrm{SH}-\mathrm{CNF}$ was smaller compared to that of CNF [23].

3.6. Thermogravimetric Analysis (TGA). TGA was used to investigate thermal stability of $\mathrm{CNF}$ and $\mathrm{SH}-\mathrm{CNF}$ samples in the temperature range of $50-500^{\circ} \mathrm{C}$ with a ramping rate of $10^{\circ} \mathrm{C} \mathrm{min}^{-1}$ (Figure 6). It could be seen that CNF and $\mathrm{SH}-\mathrm{CNF}$ showed different trend of thermal decomposition with the increase of temperature. In the initial thermal decomposition stage, the samples showed slight weight loss in the temperature range of $50-100^{\circ} \mathrm{C}$, which was mainly caused by the vaporization of a small amount of residual adsorbed water in the pores of the cellulose nanofibers. From $100^{\circ} \mathrm{C}$ to the initial decomposition temperature (at which the weight loss was 5\%), the small amount of thermal weight loss of the sample was mainly resulted from the disappearance of the hydroxyl group at the $\mathrm{C} 2$ position in the cellulose nanofiber skeleton. In the rapid thermal decomposition stage, the initial decomposition temperature to $380^{\circ} \mathrm{C}$ was the fastest process of weight loss, and the weight loss of the sample was mainly caused by the disappearance of the $\mathrm{C} 4$ hydroxyl group in the cellulose nanofiber skeleton. In the slow thermal decomposition stage, the weight loss rate of the sample gradually decreased when the temperature was raised to $400^{\circ} \mathrm{C}$, and the weight loss was mainly caused by the slow decomposition and gradual carbonization of the residues. The initial decomposition temperature of SH-CNF $\left(272^{\circ} \mathrm{C}\right)$ was higher than that of $\mathrm{CNF}\left(230^{\circ} \mathrm{C}\right)$, which resulted from $\mathrm{SH}-\mathrm{CNF}$ having a denser and firmer three-dimensional network structure after modification. At $400^{\circ} \mathrm{C}$, the residual weight of SH-CNF (39\%) was obviously higher than that of CNF (24\%). This was because silane groups grafted on SH-CNF after the modification were more stable than hydroxyl groups. The above results showed that $\mathrm{SH}-\mathrm{CNF}$ has better thermal stability than CNF after modification. 


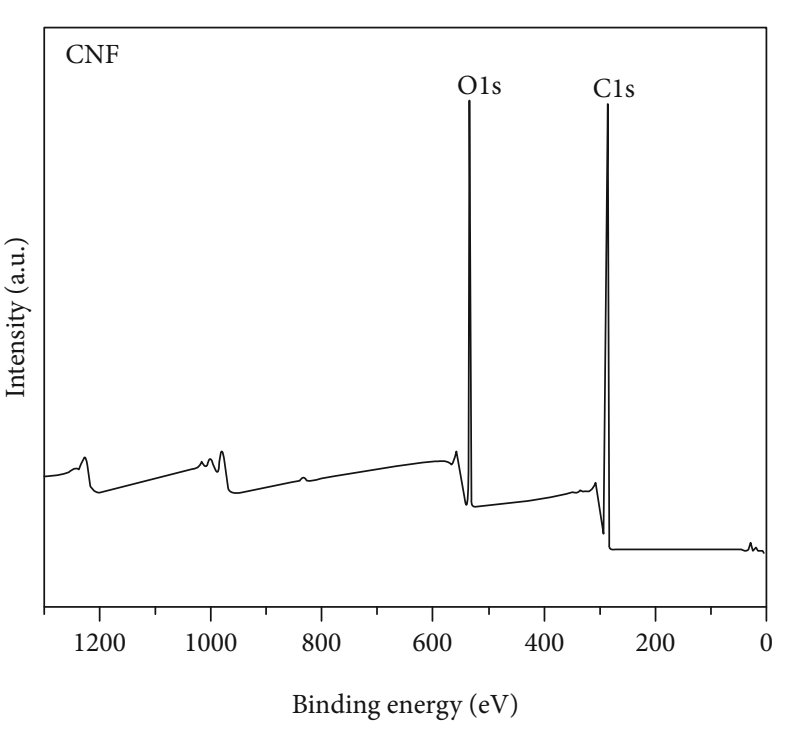

(a)

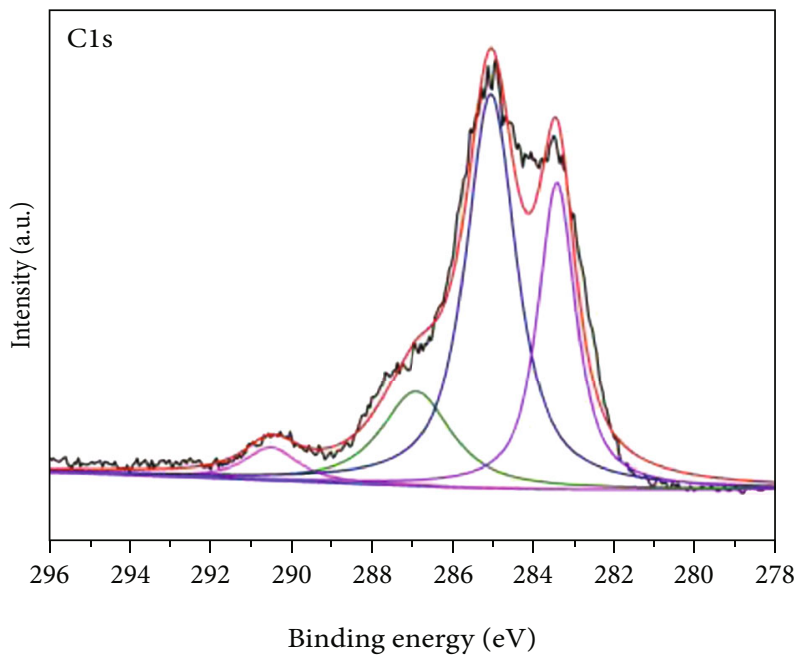

(c)

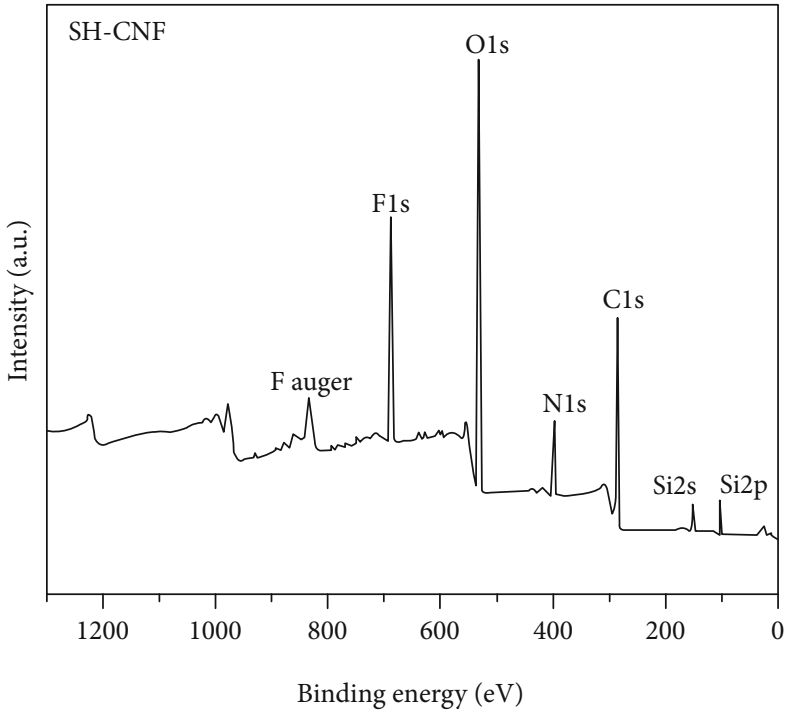

(b)

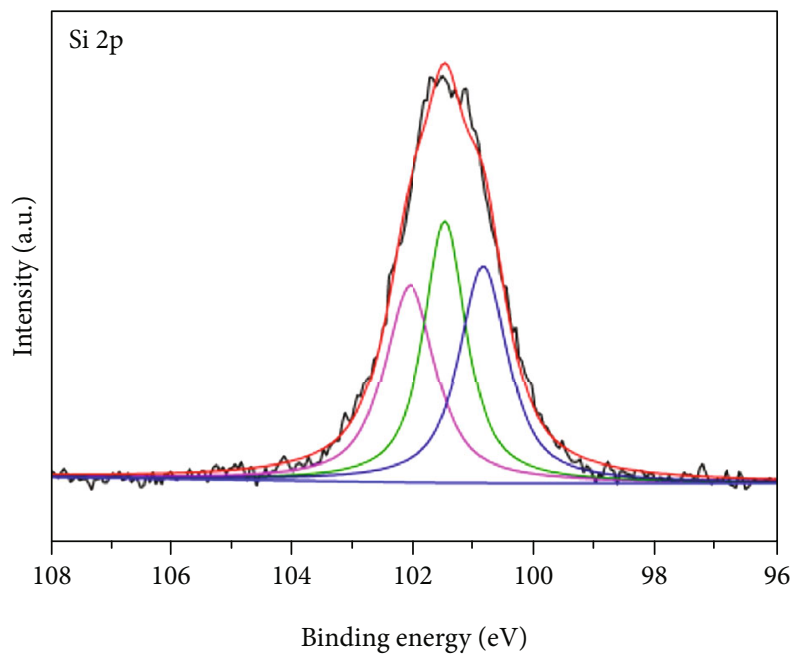

(d)

FIGURE 8: XPS survey spectra of (a) CNF and (b) SH-CNF; high resolution XPS spectra of (c) C 1s and (d) Si 2p of SH-CNF.

3.7. Scanning Electron Microscopy (SEM). Surface microstructures of $\mathrm{CNF}$ and $\mathrm{SH}-\mathrm{CNF}$ were characterized by scanning electron microscopy (Figure 7). Figures 7(c) and 7(d) show SEM images of CNF; a lot of irregular pores on the surface could be observed, but the structure was collapsed and not kept in a regular shape. Figures 7 (a) and 7(b) show the SEM images of SH-CNF, it was found that a regular array of three-dimensional layered structure was formed. The grid in the array is formed by nanofibril, closely connected in structure. The mesh size is relatively uniform. The diameter of the array and the grid is about $50 \mu \mathrm{m}$, with a larger specific surface area and porosity. This indicated that the introduction of fluorosilane and silane coupling agent had significantly changed the morphological structure of CNF. The excellent superhydrophobic property of the SH-CNF could be ascribed to the following two aspects. On the one hand, the exposed fluorosilane and silane coupling agent groups on the surface was beneficial to the decrease of surface energy. On the other hand, the modified SH-CNF surface had a micron-level array grid structure, and the water droplet on the surface was in a transition state between Wenzel and Cassie, contributing to the superhydrophobic property with a contact angle greater than $150^{\circ}[24]$.

3.8. X-Ray Photoelectron Spectroscopy (XPS). XPS was used to investigate the chemical composition of the prepared samples; the results were shown in Figure 8. Figures 8(a) and 8(b) showed the XPS survey spectra of the CNF and SH$\mathrm{CNF}$, respectively. The characteristic peaks of $\mathrm{C} 1 \mathrm{~s}$ and $\mathrm{O} 1 \mathrm{~s}$ appearing at binding energy of $285 \mathrm{eV}$ and $533 \mathrm{eV}$, respectively, were both observed through XPS survey spectra. In addition, new peaks at binding energy of 101.5, 152.1, 398.4, and $687.5 \mathrm{eV}$ were found in Figure 8(b), corresponding to $\mathrm{Si} 2 \mathrm{p}, \mathrm{Si} 2 \mathrm{~s}, \mathrm{~N}$ 1s, and F 1s, respectively. Such results indicated that $\mathrm{Si}, \mathrm{N}$, and $\mathrm{F}$ elements were added into the $\mathrm{SH}-\mathrm{CNF}$ after the modification with monomers, and the mass contents of $\mathrm{C}, \mathrm{O}, \mathrm{Si}, \mathrm{N}$, and $\mathrm{F}$ elements were $26.32 \%, 25.86 \%$, $14.96 \%, 4.48 \%$, and $28.38 \%$, respectively. 
TABLE 2: Parameters of thermal insulation property.

\begin{tabular}{lcc}
\hline Sample & $\begin{array}{c}\text { Thermal conductivity } \\
\left(\mathrm{W} \cdot \mathrm{m}^{-1} \cdot \mathrm{K}^{-1}\right)\end{array}$ & $\begin{array}{c}\text { Thermal diffusivity } \\
\left(\mathrm{mm}^{2} \cdot \mathrm{s}^{-1}\right)\end{array}$ \\
\hline CNF & 0.083 & 0.567 \\
SH-CNF & 0.035 & 0.639 \\
\hline
\end{tabular}

As shown in Figure 8(c), C 1s spectrum could be divided into four characteristic peaks at 285.1, 283.5, 286.8, and $290.3 \mathrm{eV}$, corresponding to $\mathrm{C}-\mathrm{C} / \mathrm{C}-\mathrm{H}, \mathrm{C}-\mathrm{Si}, \mathrm{C}-\mathrm{O}$, and $\mathrm{C}-\mathrm{F}$ bonds, respectively [25-27]. The $\mathrm{Si} 2 \mathrm{p}$ spectrum in Figure $8(\mathrm{~d})$ could be divided into three peaks at 100.4, 101.6 , and $102.6 \mathrm{eV}$, assigned to $\mathrm{Si}-\mathrm{C}, \mathrm{C}-\mathrm{Si}-\mathrm{O}$, and $\mathrm{Si}-\mathrm{O}$ bonds, respectively $[27,28]$. Such results further implied that the modified monomers (PFOTMS and KH540) had reacted with CNF and successfully grafted onto the surface of CNF.

3.9. Thermal Insulation Property. Laser flash diffusivity apparatus was used to investigate the thermal insulation property of $\mathrm{CNF}$ and $\mathrm{SH}-\mathrm{CNF}$, and the results were presented in Table 2. It was found that the thermal conductivity of SHCNF $\left(0.035 \mathrm{~W} \cdot \mathrm{m}^{-1} \cdot \mathrm{K}^{-1}\right)$ was obviously lower than that of the CNF $\left(0.083 \mathrm{~W} \cdot \mathrm{m}^{-1} \cdot \mathrm{K}^{-1}\right)$, indicating that the modified $\mathrm{SH}-\mathrm{CNF}$ possessed good thermal insulation performance. Combined with the results of SEM, the good thermal insulation performance of $\mathrm{SH}-\mathrm{CNF}$ could be attributed to the closely connected array three-dimensional grid structure, which was beneficial to the formation of an effective thermal resistance barrier and the decrease of convective heat transfer. On the other hand, the fluorine-silicon coating on SHCNF surface showed the thermal insulation effect and thus significantly reduced the thermal conductivity. Therefore, the superhydrophobic SH-CNF exhibited promising potential for application in the field of thermal insulation and waterproof composite materials.

\section{Conclusions}

(1) The volume ratio of modifying monomers (PFOTMS and KH540) to raw material (cellulose nanofiber emulsion) was determined to be $2 \%$, and the PFOTMS: KH540 $(v / v)$ ratio was determined as 2: 3 . The prepared modified cellulose nanofiber aerogel had a contact angle of $151^{\circ}$, which exhibited excellent superhydrophobic property

(2) The monomers (PFOTMS and KH540) were successfully grafted onto cellulose nanofibers, and the main chemical structure and crystal structure did not change obviously. The microscopic morphology of the modified cellulose nanofiber aerogels changed significantly, and the structure was more compact. It was found that a regular array three-dimensional grid structure was formed, and a superhydrophobic surface structure was successfully constructed

(3) Compared with the original cellulose nanofiber, the prepared modified cellulose nanofiber aerogel displayed better thermal stability and lower thermal conductivity, exhibiting good thermal insulation performance. The present method for the preparation of superhydrophobic cellulose nanofiber aerogels was facile and cost-effective, which laid a good theoretical foundation for their industrial production and application in the field of thermal insulation and waterproofs

\section{Data Availability}

All the data used to support the findings of this study are available from the corresponding author upon request.

\section{Conflicts of Interest}

The authors declare that they have no conflicts of interest.

\section{Acknowledgments}

This work was supported by the Basic Public Welfare Research Project of Zhejiang Province (LGG21B060001) and Science and Technology Project of Institute of Zhejiang University-Quzhou (IZQ2019-KJ-026).

\section{References}

[1] P. Panchal, E. Ogunsona, and T. Mekonnen, "Trends in advanced functional material applications of nanocellulose," Processes, vol. 7, no. 10, pp. 3390-3417, 2019.

[2] T. Guo and X. Liu, "Surface modification of cellulose nanocrystals and its reinforcing function in composite materials," Food Science, vol. 35, no. 3, pp. 285-289, 2014.

[3] D.-Y. Ye, "Preparation of nanocellulose," Progress in Chemistry, vol. 19, no. 10, pp. 1568-1574, 2007.

[4] J.-C. Wittmanm and P. Smith, "Highly oriented thin films of poly(tetrafluoroethylene) as a substrate for oriented growth of materials," Nature, vol. 352, no. 6334, pp. 414-417, 1991.

[5] S. Ifuku, M. Nogi, K. Abe, K. Handa, F. Nakatsubo, and H. Yano, "Surface modification of bacterial cellulose nanofibers for property enhancement of optically transparent composites dependence on acetyl-droup," Biomacromolecules, vol. 8, no. 6, pp. 1973-1978, 2007.

[6] J. Zhang, W. Chen, Y. Feng et al., "Homogeneous esterification of cellulose in room temperature ionic liquids," Polymer International, vol. 64, no. 8, pp. 963-970, 2015.

[7] N. Wang, E.-Y. Ding, and R.-S. Cheng, "The surface modification of nanocrystaline cellulose," Acta Polymerica Sinica, vol. 8, pp. 982-987, 2006.

[8] M. Zhang and C. Wang, "Fabrication of cotton fabric with superhydrophobicity and flame retardancy," Carbohydrate Polymers, vol. 96, no. 2, pp. 396-402, 2013.

[9] Z. Gao, X. Zhai, F. Liu, M. Zhang, D. Zang, and C. Wang, "Fabrication of $\mathrm{TiO}_{2} / \mathrm{EP}$ super-hydrophobic thin film on filter paper surface," Carbohydrate Polymers, vol. 128, pp. 24-31, 2015.

[10] S. Zhang, W. Li, Y. Yang et al., "Preparation and characterization of reactive superhydrophobic surface based on cellulosederived micro/nanoparticle," Transactions of China Pulp and Paper, vol. 35, no. 2, pp. 23-28, 2020.

[11] Z.-D. Zhu, X.-M. Zheng, S.-Y. Fu, and W.-Y. Zhu, "Effect of silane modified cellulosic micro-nano particles on super- 
hydrophobicity of material," China Pulp \& Paper, vol. 37, no. 12, pp. 14-20, 2018.

[12] Z. Zhang, G. Sèbe, D. Rentsch, T. Zimmermann, and P. Tingaut, "Ultralightweight and flexible silylated nanocellulose sponges for the selective removal of oil from water," Chemistry of Materials, vol. 26, no. 8, pp. 2659-2668, 2014.

[13] M. Abdelmouleh, S. Boufi, M.-N. Belgacem, A. P. Duarte, A. Ben Salah, and A. Gandini, "Modification of cellulosic fibres with functionalised silanes: development of surface properties," International Journal of Adhesion and Adhesives, vol. 24, no. 1, pp. 43-54, 2004.

[14] M. Abdelmouleh, S. Boufi, A. ben Salah, M. N. Belgacem, and A. Gandini, "Interaction of silane coupling agents with cellulose," Langmuir, vol. 18, no. 8, pp. 3203-3208, 2002.

[15] M. Castellano, A. Gandini, P. Fabbri, and M. N. Belgacem, "Modification of cellulose fibres with organosilanes: under what conditions does coupling occur?," Journal of Colloid and Interface Science, vol. 273, no. 2, pp. 505-511, 2004.

[16] L. Fan, Y.-B. Weng, P.-T. Liu, and Z. Liu, "Preparation and characterization of hydrophobic cellulose nanofibril aerogels," Journal of Functional Materials, vol. 51, no. 2, pp. 0210702112, 2020.

[17] N.-T. Cervin, C. Aulin, P.-T. Larsson, and L. Wågberg, "Ultra porous nanocellulose aerogels as separation medium for mixtures of oil/water liquids," Cellulose, vol. 19, no. 2, pp. 401-410, 2012.

[18] J. Huang, S. Wang, S. Lyu, and F. Fu, "Preparation of a robust cellulose nanocrystal superhydrophobic coating for selfcleaning and oil-water separation only by spraying," Industrial Crops and Products, vol. 122, pp. 438-447, 2018.

[19] M. M. Bashar, H. Zhu, S. Yamamoto, and M. Mitsuishi, "Superhydrophobic surfaces with fluorinated cellulose nanofiber assemblies for oil-water separation," RSC Advances, vol. 7, no. 59, pp. 37168-37174, 2017.

[20] C.-H. Xue, S.-T. Jia, J. Zhang, and L. Q. Tian, "Superhydrophobic surfaces on cotton textiles by complex coating of silica nanoparticles and hydrophobization," Thin Solid Films, vol. 517, no. 16, pp. 4593-4598, 2009.

[21] A. Salam, L.-A. Lucia, and H. Jameel, "Fluorine-based surface decorated cellulose nanocrystals as potential hydrophobic and oleophobic materials," Cellulose, vol. 22, no. 1, pp. 397406, 2015.

[22] M. Mohkami and M. Talaeipour, "Investigation of the chemical structure of carboxylated and carboxymethylated fibers from waste paper via XRD and FTIR analysis," BioResources, vol. 6, no. 2, pp. 1988-2003, 2011.

[23] H. L. Liu, J. J. Peng, M. Li, and F. Yang, "Preparation and photocatalytic activity of $\mathrm{TiO}_{2}$ /nanocrystalline cellulose composite films coating on glass," Acta Materiae Compositae Sinica, vol. 30, no. 4, pp. 163-169, 2013.

[24] J. M. Wang, C. Wang, M. C. Wang, and L. Jiang, "Effects of distribution of microconcave array with nanofolds on the water adhesive property of PDMS films," Chemical Journal of Chinese Universities, vol. 33, no. 10, pp. 2333-2338, 2012.

[25] J. Yang, L. Cao, R. Guo, and J. Jia, "Permeable reactive barrier of surface hydrophobic granular activated carbon coupled with elemental iron for the removal of 2,4-dichlorophenol in water," Journal of Hazardous Materials, vol. 183, no. 1-3, pp. 782-787, 2010.

[26] C. T. Hsieh, J. M. Chen, Y. H. Huang et al., "Influence of fluorine/carbon atomic ratio on superhydrophobic behavior of car- bon nanofiber arrays," Journal of Vacuum Science \& Technology B, vol. 24, no. 1, pp. 113-117, 2006.

[27] Y. Kazuhiro, K. Yoshinori, and F. Shuzo, "XPS studies of amorphous SiCN thin films prepared by nitrogen ionassisted pulsed-laser deposition of SiC target," Diamond and Related Materials, vol. 10, no. 9-10, pp. 1921-1926, 2001.

[28] M. Veres, M. Koós, S. Tóth et al., "Characterisation of a-C:H and oxygen-containing Si:C:H films by Raman spectroscopy and XPS," Diamond and Related Materials, vol. 14, no. 3-7, pp. 1051-1056, 2005. 